\title{
Wide cross-species RNA-Seq comparison reveals a highly conserved role for Ferroportins in nickel hyperaccumulation in plants
}

Vanesa S. Garcia de la Torre ${ }^{1}$, Clarisse Majorel-Loulergue ${ }^{2}$, Dubiel A. Gonzalez ${ }^{3}$, Ludivine Soubigou-Taconnat ${ }^{4,5}$, Guillem J. Rigaill ${ }^{4,6}$, Yohan Pillon ${ }^{7}$, Louise Barreau ${ }^{1}$, Sébastien Thomine $^{1}$, Bruno Fogliani ${ }^{8}$, Valérie Burtet-Sarramegna ${ }^{2}$, Sylvain Merlot ${ }^{1^{*}}$

${ }^{1}$ Institute for Integrative Biology of the Cell (I2BC), CEA, CNRS, Univ. Paris-Sud, Université Paris-Saclay, 91198, Gif-sur-Yvette cedex, France

${ }^{2}$ Institute of Exact and Applied Sciences (ISEA), Université de la Nouvelle-Calédonie, BP R4, 98851, Nouméa cedex, New Caledonia

${ }^{3}$ Universidad Agraria de La Habana (UNAH), Departamento de Biologia, 32700, Mayabeque, Cuba

${ }^{4}$ Institute of Plant Sciences Paris-Saclay (IPS2), CNRS, INRA, Université Paris-Sud, Université d'Evry, Université Paris-Saclay, Université Paris-Diderot, Sorbonne Paris-Cité, Bâtiment 630, Plateau du Moulon, 91192, Gif-sur-Yvette cedex, France

${ }^{5}$ Institute of Plant Sciences Paris-Saclay (IPS2), CNRS, INRA Université Paris-Diderot, Sorbonne Paris-Cité, Bâtiment 630, Plateau du Moulon, 91192, Gif-sur-Yvette cedex, France

${ }^{6}$ Laboratoire de Mathématiques et Modélisation d'Evry (LaMME), Université d'Evry, CNRS, ENSIIE, USC INRA, 23 bvd de France, 91037, Evry cedex, France

${ }^{7}$ Laboratoire des Symbioses Tropicales et Méditerranéennes (LSTM), IRD, INRA, CIRAD, Montpellier SupAgro, Univ. Montpellier, 34398, Montpellier cedex, France

${ }^{8}$ Institut Agronomique néo-Calédonien (IAC), Equipe ARBOREAL, BP 73, 98890, Païta, New Caledonia

${ }^{*}$ Corresponding author: sylvain.merlot@i2bc.paris-saclay.fr, Phone: +33-169824642

Introductory paragraph: 151 words

Manuscript: 1388 words

1 Table

3 Main Figures

5 Supplementary Figures

11 Supplementary Data files 
The Anthropocene epoch is associated with the spreading of metals in the environment increasing oxidative and genotoxic stress on living organisms $s^{1,2}$. Once regarded as a curiosity, plants hyperaccumulating metals are now envisioned as an opportunity to remediate metal contaminated soils. About 500 plant species adapted to metalliferous soils acquired the capacity to hyperaccumulate $(>0.1 \%$ of dry weight) nickel in their shoot $^{3}$. The phylogenetic distribution of these hyperaccumulators in 50 families suggest that this complex trait evolved multiple times independently from basic mechanisms involved in metal homeostasis. However, the exact nature of these mechanisms and whether they are shared between various lineages is not known. Here, using cross-species transcriptomic analyses in different plant families, we have identified convergent functions that may represent major nodes in the evolution of nickel hyperaccumulation. In particular, our data point out that constitutive high expression of IREG/Ferroportin transporters recurrently emerged as a mechanism involved in nickel hyperaccumulation.

Nickel hyperaccumulators are found worldwide as herbaceous plants, shrubs or trees growing on outcrops originating from ultramafic rocks, such as serpentine soils rich in iron, nickel, and cobalt. Although nickel hyperaccumulation is a rare and complex trait, it appeared independently in many distant plant families worldwide (Figure 1). In this study, we ask whether the same molecular mechanisms have been recruited convergently to reach this extreme trait. To address this question, we first compared the transcriptomes of nickel hyperaccumulators and closely related non-accumulator species to identify candidate genes involved in nickel hyperaccumulation. Then, in a second step, we established orthologous relationships to identify genes with similar functions that display differential expression in nickel hyperaccumulators from distant families.

We harvested leaves of 7 nickel hyperaccumulators from 5 genera (i.e, Noccaea, Psychotria, Geissois, Homalium, Leucocroton) corresponding to distant plant families in their natural environment in France, New Caledonia and Cuba (Figure 1, Table 1). For each 
hyperaccumulator, we collected leaves of a closely related species or ecotype not accumulating nickel in the same geographic area. To identify genes differentially expressed between closely related nickel hyperaccumulator and non-accumulator species, we performed RNA sequencing (RNA-Seq) using Illumina paired-end technology (Supplementary Data 2). In absence of genomic sequence references for most of these species, we de novo assembled the short Illumina reads into the sequence of expressed genes in the 13 selected species. These newly generated transcriptomes contain between 41,843 and 87,243 contigs for a total assembly size ranging from $35 \mathrm{Mbp}$ to $49 \mathrm{Mbp}$ (Table 1, Supplementary Data 2). Subsequent annotation of these transcriptomes using Blastx interrogation of Viridiplantae proteins database revealed significant homologies for $56 \%$ of the contigs on average (E-value $\leq 10 \mathrm{E}-6)$. These high-quality reference transcriptomes constitute a unique and comprehensive resource for molecular studies on plant groups of high interest.

The reads from each sample were then mapped to the nickel hyperaccumulator reference transcriptome to identify differentially expressed (DE) genes in 8 pairs of species, each containing a hyperaccumulator and a related non-nickel accumulator. The fraction of DE genes ranged from $2 \%$ to $29 \%$ (Fold Change $\geq 2, F D R \leq 0.05$ ), depending on the pair of species considered (Figure 2a, Supplementary Figure 2 and Supplementary Data 4-9). Swapping to the transcriptome of the non-accumulators as reference had only a marginal influence on the results (Supplementary Figure 3).

To determine if deregulated molecular and metabolic pathways are shared by nickel hyperaccumulators from distant plant families, it is necessary to compare these transcriptomes together with a functional emphasis. To this aim, we classified the gene products into Clusters of Orthologous Groups (COG). Combining the 13 reference transcriptomes, 443,400 contigs (66.5\% of total) were assigned to 46,458 COGs (Supplementary Data 3).

To target genes involved in nickel hyperaccumulation, we looked for COGs containing differentially expressed genes in at least 3 plant families. This analysis revealed 71 groups, of which 33 COGs included orthologous genes more expressed in nickel hyperaccumulators, 15 COGs included orthologous genes less expressed and 22 COGs were heterogeneous with 
some orthologs more expressed and some orthologs less expressed (Supplementary Data 10).

The functional annotation of these COGs (Figure $\mathbf{2 b}$ ) indicated that the most represented category corresponds to genes involved in the biosynthesis of secondary metabolites, more specifically phenylpropanoids and flavonoids molecules (Supplementary Figure 4). The analysis of gene expression in these pathways predicts an increased synthesis of flavonoids in leaves of nickel hyperaccumulators. This suggests an unsuspected role for flavonoids in nickel hyperaccumulation. These molecules have been shown to act as antioxidants or directly as metal ligands, including nickel, in vitro ${ }^{5,6}$. Furthermore, it has been recently shown that flavonoids accumulate in an Arabidopsis halleri population hyperaccumulating cadmium ${ }^{7}$. Further metabolomics, speciation and genetics studies will be required to establish the importance of flavonoids in nickel hyperaccumulation.

Previous studies have highlighted the important role of histidine in nickel hyperaccumulation in Brassicaceae ${ }^{8}$. Our data reveal that orthologs of histidinol dehydrogenase (COG5550), catalyzing the last step of histidine synthesis, are more expressed in nickel hyperaccumulators from Rubiaceae, Cunoniaceae and Euphorbiaceae families supporting the role of histidine in nickel hyperaccumulation (Figure 2c). Interestingly, in the hyperaccumulator Alyssum lesbiacum of the Brassicaceae family, the first step of histidine biosynthesis (ATP-PRT) is upregulated $^{9}$. This result suggests that distinct steps of the histidine biosynthetic pathway are amplified converging to increased histidine synthesis in nickel hyperaccumulators from different families.

Our cross-species comparison points to IREG/Ferroportin transporters (COG1981) as the most robust up-regulated function among distantly related nickel hyperaccumulators (Figure 2c, Supplementary Data 10). IREG/Ferroportin (SLC40) are efflux transporters found in different kingdoms and displaying broad specificity for divalent metal ions ${ }^{10,11}$. In vertebrates, the main function of IREG/Ferroportin is to release iron from cells $s^{12-14}$. In plants, IREG/Ferroportin transporters have been associated with the regulation of metal homeostasis including iron, nickel, cobalt and aluminum ${ }^{15-19}$. Genes encoding IREG/Ferroportin 
transporters are significantly more expressed (from 4 to 800 -fold increase) in nickel hyperaccumulators from the Brassicaceae, Rubiaceae and Euphorbiaceae families compared to their related non-accumulator species. In Salicaceae and Cunoniaceae species from New Caledonia, IREG/Ferroportin genes are highly expressed in both hyperaccumulators and nonaccumulators (Supplementary Figure 5a). However, the unique IREG/Ferroportin gene (COG1981) detected in the hyperaccumulator Geissois pruinosa is 60-times more expressed than its orthologue in the non-accumulator Cunoniaceae species Cunonia capensis from South Africa (Supplementary Figure 5b). Therefore, the high expression of IREG/Ferroportin genes in non-accumulator species endemic from New Caledonia might be a genetic footprint of the recent colonization ( $\sim 35 \mathrm{MYA}$ ) of this island then probably fully covered by an ultramafic rock layer ${ }^{20,21}$.

To provide functional evidence for the role of plant IREG/Ferroportin in nickel hyperaccumulation, we expressed IREG/Ferroportin orthologs cloned from 3 distant nickel hyperaccumulator species in yeast (Figure 3a). The expression of these transporters increases yeast resistance to nickel, which is consistent with a conserved activity of plant IREG/Ferroportin as nickel exporters. Then we investigated the biological relevance of IREG/Ferroportin in nickel hyperaccumulation in planta. We used Rhizobium rhizogenes transformation to silence the expression of NcIREG2 in the roots of the nickel hyperaccumulator $N$. caerulescens subsp. firmiensis using artificial miRNA technology. We chose to target NcIREG2 which shows strong differential expression in leaves and roots associated with nickel hyperaccumulation in Noccaea caerulescens (this study and ${ }^{22}$ ). We generated 8 independent transgenic lines displaying different degrees of NcIREG2 silencing (Figure 3b). Elemental analysis of these transgenic lines revealed a decrease of nickel accumulation in roots. Moreover, the amplitude of nickel accumulation strongly correlates with NcIREG2 expression (Pearson's $r=0.9, \mathrm{p}$-value $=5.3 \mathrm{E}-5)$. To understand the cellular basis for NcIREG2 mediated nickel accumulation, we expressed a GFP tagged version of this transporter in roots. Confocal imaging of transgenic roots shows that NcIREG2-GFP localizes 
on the membrane of the vacuole. Together, our results provide genetic evidence that NcIREG2 contributes to nickel hyperaccumulation by driving nickel sequestration in vacuoles.

The development of RNA-Seq technologies has opened the possibility to study non-model species at the molecular level. Yet, comparative biology has not fully benefited from this revolution because of the difficulty to quantitatively compare transcriptomes from distant species. In this study, we have used a combination of cross-species comparative transcriptomics analysis and COG annotation to identify genes associated with nickel hyperaccumulation in a wide diversity of plant families. This analysis revealed a limited number of candidate gene functions corresponding to convergent mechanisms involved in nickel hyperaccumulation including flavonoid and histidine biosynthesis, and nickel transport. Strikingly, constitutive high expression of IREG/Ferroportin in leaves has been recurrently recruited as a convergent mechanism for nickel hyperaccumulation. Our functional analysis provides evidence that these transporters account for the exceptional ability of hyperaccumulators to store nickel in vacuoles. This shows that our transcriptomic approach predicts important actors in nickel hyperaccumulation. This study further highlights other candidate genes as target for future functional analyses. Moreover, it provides a framework to identify key genes from root transcriptomes that are involved in the efficient uptake and translocation of nickel to the leaves. The identification of all molecular steps from uptake in roots to sequestration in leaves is necessary to fully understand this complex trait. In the context of sustainable development, nickel hyperaccumulators are now viewed as crops to extract and recycle metals from large areas of metalliferous soils ${ }^{23,24}$. As for other crops, we foresee that this molecular knowledge could become instrumental for marker-assisted selection of cultivars or molecular monitoring of agricultural practices to improve nickel phytoextraction.

METHODS. Methods, including statements of data availability and associated accession codes and references, are available in the online version of the paper.

Note: Any Supplementary Information is available in the online version of the paper 
ACKNOWLEDGMENTS. We thank professor Rosalina Berazaín Iturralde (UNAH, Cuba) for invaluable information on Cuban flora, Louis-Charles Brinon (IAC, New Caledonia) for sample collection in New Caledonia, Véronique Brunaud and Marie-Laure Martin-Magniette (IPS2, France) for guidance on de novo assembly and biostatistical analysis, and Mark G. M. Aarts (WUR, Netherlands) for the Noccaea root transformation protocol.

This work was supported by Grants ANR-13-ADAP-0004 (SM, BF, VBS) and CNRS Defi Enviromics Gene-4-Chem to SM, a SCAC fellowship from the French Embassy in Cuba to DAGand SM and an ATIGE grant from Génopole to GJR. The I2BC and POPS platform benefit from the support of the LabEx Saclay Plant Sciences-SPS (ANR-10-LABX-0040-SPS). This work has benefited from the core facilities of Imagerie-Gif, a member of Infrastructures en Biologie Santé et Agronomie (IBiSA), supported by France Biolmaging Grant ANR-10INBS04-01 and the Saclay Plant Science Labex Grant ANR-11-IDEX-0003-0. We thank the South Province of New Caledonia and the Prefecture of Aveyron for plant collection authorizations.

AUTHOR CONTRIBUTIONS. BF, VBS and SM designed the project; VSG, CML, DAG, BF, VBS and SM collected plant samples; VSG, CML, DAG, LB performed experiments; LST supervised RNA-Seq sequencing; VSG, GJR, YP, ST and SM analyzed the data; VSG, ST and SM wrote the manuscript; all authors commented and approved the content of the manuscript.

COMPETING FINANTIAL INTERESTS. The authors declare no competing financial interests.

1. Monge, G. et al. Earliest evidence of pollution by heavy metals in archaeological sites. Sci. Rep. 5, 1-9 (2015).

2. McConnell, J. R. \& Edwards, R. Coal burning leaves toxic heavy metal legacy in the Arctic. Proc. Natl. Acad. Sci. USA 105, 12140-12144 (2008). 
3. Reeves, R. D. et al. A global database for plants that hyperaccumulate metal and metalloid trace elements. New Phytol. 218, 407-411 (2018).

4. Magallón, S., Gómez-Acevedo, S., Sánchez-Reyes, L. L. \& Hernández-Hernández, T. A metacalibrated time-tree documents the early rise of flowering plant phylogenetic diversity. New Phytol. 207, 437-453 (2015).

5. Pietta, P.-G. Flavonoids as Antioxidants. J. Nat. Prod. 63, 1035-1042 (2000).

6. Kasprzak, M. M., Erxleben, A. \& Ochocki, J. Properties and applications of flavonoid metal complexes. RSC Adv. 5, 45853-45877 (2015).

7. Corso, M. et al. Contrasting cadmium resistance strategies in two metallicolous populations of Arabidopsis halleri. New Phytol. 218,283-297 (2018).

8. Krämer, U., Cotter-Howells, J. D., Charnock, J. M., Baker, A. J. M. \& Smith, J. A. C. Free histidine as a metal chelator in plants that accumulate nickel. Nature $\mathbf{3 7 9}, 635-638$ (1996).

9. Ingle, R. A., Mugford, S. T., Rees, J. D., Campbell, M. M. \& Smith, J. A. Constitutively high expression of the histidine biosynthetic pathway contributes to nickel tolerance in hyperaccumulator plants. Plant Cell 17, 2089-2106 (2005).

10. Taniguchi, R. et al. Outward and inward facing structures of a putative bacterial transition metal transporter with homology to ferroportin. Nat. Commun. 6, 1-10 (2015).

11. Madejczyk, M. S. \& Ballatori, N. The iron transporter ferroportin can also function as a manganese exporter. Biochim. Biophys. Acta - Biomembr. 1818, 651-657 (2012).

12. Abboud, S. \& Haile, D. J. A novel mammalian iron-regulated protein involved in intracellular iron metabolism. J. Biol. Chem. 275, 19906-19912 (2000).

13. Donovan, A et al. Positional cloning of zebrafish ferroportin1 identifies a conserved vertebrate iron exporter. Nature 403, 776-781 (2000).

14. McKie, A. et al. A novel duodenal iron-regulated transporter, IREG1, implicated in the basolateral transfer of iron to the circulation. Mol. Cell 5, 299-309 (2000). 
15. Schaaf, G. et al. AtIREG2 encodes a tonoplast transport protein involved in irondependent nickel detoxification in Arabidopsis thaliana roots. J. Biol. Chem. 281, 2553240 (2006).

16. Morrissey, J. et al. The ferroportin metal efflux proteins function in iron and cobalt homeostasis in Arabidopsis. Plant Cell 21, 3326-38 (2009).

17. Conte, S., Stevenson, D., Furner, I. \& Lloyd, A. Multiple antibiotic resistance in Arabidopsis is conferred by mutations in a chloroplast-localized transport protein. Plant Physiol. 151, 559-573 (2009).

18. Merlot, S. et al. The metal transporter PgIREG1 from hyperaccumulator Psychotria gabriellae is a candidate gene for nickel tolerance and accumulation. J. Exp. Bot. 65, $1551-1564(2014)$.

19. Yokosho, K., Yamaji, N., Mitani-ueno, N., Shen, R. F. \& Ma, J. F. An Aluminum-inducible IREG gene is required for internal detoxification of aluminum in buckwheat. Plant Cell Physiol. 57, 1169-1178 (2018).

20. Pillon, Y., Munzinger, J., Amir, H. \& Lebrun, M. Ultramafic soils and species sorting in the flora of New Caledonia. J. Ecol. 98, 1108-1116 (2010).

21. Pillon, Y., Hopkins, H. C. F., Rigault, F., Jaffré, T. \& Stacy, E. A. Cryptic adaptive radiation in tropical forest trees in New Caledonia. New Phytol. 202, 521-530 (2014).

22. Halimaa, P. et al. Gene expression differences between Noccaea caerulescens ecotypes help to identify candidate genes for metal phytoremediation. Environ. Sci. Technol. 48, 3344-53 (2014).

23. Van Der Ent, A. et al. Agromining: Farming for metals in the future? Environ. Sci. Technol. 49, 4773-4780 (2015).

24. Losfeld, G., L'Huillier, L., Fogliani, B., Jaffré, T. \& Grison, C. Mining in New Caledonia: environmental stakes and restoration opportunities. Environ. Sci. Pollut. Res. Int. 22(8), $5592-607$ (2015) 


\section{ONLINE METHODS}

Plant material and sample collection. Leaves of nickel hyperaccumulator plants belonging to 5 distinct plant families ${ }^{25-28}$, Brassicaceae [Noccaea caerulescens subsp. firmensis (Ncfi)], Cunoniaceae [Geissois pruinosa (Gpru)], Euphorbiaceae [Leucocroton havanensis (Lhav)], Rubiaceae [P. costivenia (Pcos), P. gabriellae (Pgab), Psychotria grandis (Pgra)], Salicaceae [Homalium kanaliense (Hkan)], and of related nonaccumulator species, Brassicaceae [N. caerulescens "Viviez" (Ncviv), N. montana (Nmon)], Cunoniaceae [G. racemosa (Grac)], Euphorbiaceae [Lasiocroton microphyllus (Lmic)], Rubiaceae [P. clementis (Pcle), P. semperflorens (Psem)], Salicaceae [H. betulifolium (Hbet)], were collected from individual plants growing in their natural environment in France, New Caledonia and Cuba (Supplementary Data 1). We complied with local regulation for the access to these genetic resources. Each sample was localized by GPS. For each sample, a fraction of leaves was washed with water and dried for elemental analysis, and the other fraction fixed on site with liquid $\mathrm{N}_{2}$ (Gpru, Grac, Pgab, Psem) or with RNAlater (Sigma Aldrich) and stored at $4^{\circ} \mathrm{C}$ (Ncfi, Ncviv, Nmon, Lhav, Lmic, Pcos, Pgra, Pcle, Hkan, Hbet). RNAlater was removed in the laboratory and leaves were immediately stored at $-80^{\circ} \mathrm{C}$ before RNA extraction.

Additionally, to generate reference transcriptomes, Ncfi and Nmon were grown in hydroponic condition using a modified Hoagland's solution ${ }^{29}$ containing $20 \mu \mathrm{M}$ Fe-HBED (Van Iperen International) and $37.5 \mu \mathrm{M} \mathrm{NiCl}_{2}$, in a climatic chamber (9 h light, $150 \mu \mathrm{E} \mathrm{m} \mathrm{m}^{-}$ ${ }^{2} \mathrm{~s}^{-1}, 21^{\circ} \mathrm{C} / 17^{\circ} \mathrm{C}$ day/night; $70 \%$ humidity) for 7 weeks. L. havanensis seeds were cultured in vitro on Murashige \& Skoog Agar medium supplemented with $3.2 \mathrm{mM} \mathrm{NiSO}_{4}$, as described ${ }^{30}$.

A specimen of Cunonia capensis (Ccap) was obtained from a specialized plant nursery (Ets. Railhet, France) and grown in a green-house on coconut fiber supplemented with fertilizer.

RNA sequencing. Total RNA from leaves were extracted with RNeasy Plant Mini kit 
(Qiagen) for Noccaea species, Qiagen hybrid method for woody plants ${ }^{31}$ for Psychotria species from Cuba, CTAB-PVP method ${ }^{31}$ for Geissois and Psychotria species from New Caledonia, and TRI Reagent (Sigma-Aldrich) for Leucocroton, Lasiocroton and Homalium species. DNA was removed from all RNA samples by RNeasy Plant Mini kit on-column DNase I treatment.

RNA quality control, preparation of cDNA libraries, sequencing and raw reads processing were performed by the POPS transcriptomic platform (IPS2, Orsay, France). Libraries were prepared from $1 \mu \mathrm{g}$ of total RNA using TruSeq Stranded mRNA kit (Illumina) and sequenced with an Illumina HiSeq2000 sequencing system in 100bp paired-end mode. Libraries meant to be directly compared were multiplexed and sequenced in a single run. Adaptors and low-quality pair-end sequences were removed from the raw reads and the ribosomal RNA was filtered using the SortMeRNA algorithm ${ }^{32}$. We obtained between 27 and 106 million reads per libraries (Supplementary Data 2).

De novo transcriptome assembly and annotation. The transcriptome sequences of Ncfi, Nmon, Pgab, Psem, Pgra, Pcos, Pcle, Gpru, Grac, Hkan, Hbet, Lhav and Lmic were obtained independently by de novo assembly of paired-end reads using CLC Genomics Workbench v9 software (Qiagen). A single library per species was used to minimize genetic variability. Assembly parameters were set as default, except similarity (0.95), length fraction (0.75) and the word size was optimized for each sample (Supplementary Data 2). The sequences of the resulting contigs were blasted (Blastx, E-value of $\leq 10 \mathrm{E}-$ 6) against the Viridiplantae protein database (NCBI) and putative function annotated by Gene Onthology (cut-off $=55 ; \mathrm{GO}$ weight $=-5$ ) using Blast2GO${ }^{33}$. Filtered contigs for length ( $\geq 200 \mathrm{nt})$ and expression (TPKM > 1) were translated for the longest Open Reading Frame. Translated sequences longer than 20 amino acids, together with Arabidopsis thaliana protein sequences (TAIR10, www.arabidopsis.org) were analyzed by OrthoFinder ${ }^{34}$ to annotate Clusters of Orthologous Group (COG). 
Differential gene expression analysis. For each pair of species, read count estimation was carried out using CLC Genomics Workbench v9 software by mapping sequencing reads of each sample (default parameters, except similarity: 0.875 and length fraction: $0.75)$ to the transcriptome of the nickel hyperaccumulator species used as the reference. Statistical analyses to identify Differentially Expressed Genes (DEGs) were performed using the edgeR Bioconductor package ${ }^{35}$. To examine transcript abundance, reads per kilobase million (RPKM) were calculated. To evaluate the influence of the reference transcriptome, we additionally performed swapping analyzes using the transcriptome of the non-accumulator species as reference (Supplementary Data 2, Supplementary Figure 3).

Molecular cloning. Predicted full-length coding region of NcfilREG2, GpruIREG1 and LhavIREG2 were amplified from leaf cDNAs of the corresponding species, using highfidelity Phusion polymerase (Thermo Scientific) with gene specific primers containing AttB recombination sequences (Supplementary Data 11). PCR products were first recombined into pDONOR207 (Invitrogen) and then in pDR195-GTW ${ }^{36}$ or pMDC83 ${ }^{37}$ for expression in yeast and $N$. caerulescens respectively.

Artificial miRNA construct targeting NcfilREG2 was designed using the WMD3-Web microRNA Designer (http://wmd3.weigelworld.org). The amiRNA was engineered as previously described ${ }^{38}$ by PCR using the pRS300 backbone and specific primers (Supplementary Data 11). The NcfilREG2-amiRNA precursor was recombined in pDONOR207 and then into the vector pK7GW2D ${ }^{39}$.All constructs were confirmed by restriction analysis and sequencing.

Functional analyses of IREG/Ferroportin in yeast. IREG/ferroportin coding regions cloned into pDR195-GTW, as well as pDR195-AtIREG $2^{15}$, were transformed by the lithium acetate method into the Saccharomyces cerevisiae BY4741 strain complemented by a functional HIS3 gene. Yeast sensitivity to nickel was scored by yeast drop assay 
using serial dilution on histidine-free YNB agar medium containing $20 \mathrm{mM}$ MES (pH5.5), supplemented or not with $\mathrm{NiCl}_{2}$. Empty pDR195 vector was used as control. Experiments were repeated twice with three independent transformants.

Functional analysis of NcfilREG2 in transgenic plants. pK7GW2D-NcfilREG2amiRNA and pMDC83-NcfilREG2 were transformed into $N$. caerulescens subsp. firmiensis by Rhizobium rhizogenes (Arqua1 strain) mediated in vitro root transformation ${ }^{40}$. Transformed roots were selected using GFP fluorescence under a Leica MZ FLIII Fluorescence Stereo Microscope. Non-transformed roots were cut once a week until the whole root system was transgenic.

Independent lines transformed with pK7GW2D-NcIREG2-amiRNA were then transferred in hydroponic culture as described above for a week and then with the nutrient solution supplemented with $37.5 \mu \mathrm{M} \mathrm{NiCl}_{2}$ for 4 weeks. For each transgenic line, the root system was divided in two samples for RT-qPCR and elemental analyzes.

Elemental analysis. Dry Environmental leaf samples were mineralized by $\mathrm{HNO}_{3}$. Multielemental analyzes were performed using ICP-AES (LAMA laboratory, IRD, New Caledonia) or MP-AES.

Transgenic root samples were washed twice with ice-cold $10 \mathrm{mM} \mathrm{Na}{ }_{2}$ EDTA and twice with ice-cold ultrapure water. Samples were dried at $65^{\circ} \mathrm{C}$ for 16 hours, weighted and then digested with $70 \% \mathrm{HNO}_{3}$ and $\mathrm{H}_{2} \mathrm{O}_{2}$ for a total of $8 \mathrm{~h}$ with temperature ramping from 80 to $120^{\circ} \mathrm{C}$. Elemental analyzes were performed using MP-AES (Agilent 4200, Agilent Technologies) and metal concentration was calculated by comparison with a metal standard solution.

Confocal Imaging. Root transformed with pMDC83-NcfilREG2 were stained with 10 $\mu \mathrm{g} / \mathrm{ml}$ propidium iodide $(\mathrm{PI})$ and imaged on a Leica SP8X inverted confocal microscope 
(IMAGERIE-Gif platform) with laser excitation at $488 \mathrm{~nm}$ and collection of emitted light at $495-550 \mathrm{~nm}$ for GFP and $600-650 \mathrm{~nm}$ for PI.

Quantitative RT-PCR analyzes. Total RNA from Ncfi transgenic roots were extracted with TRI Reagent (Sigma-Aldrich). Total RNA from leaves of Gpru and Ccap were extracted with the CTAB-PVP method as described above. DNA was removed from all RNA samples by RNeasy Plant Mini kit on-column DNase I treatment. RNA (1 $\mu \mathrm{g})$ were converted to cDNA by random priming using SuperScript III First-Strand reverse transcriptase (Invitrogen) according to the manufacturer's instructions. Quantitative PCR analysis were performed on a LightCycler 96 using LightCycler 480 SYBR Green I Master Mix (Roche) with the following conditions: Initial denaturation (95 $\left.{ }^{\circ} \mathrm{C}, 300 \mathrm{~s}\right)$, followed by 40 cycles of amplification $\left(95^{\circ} \mathrm{C}, 15 \mathrm{~s} ; 60{ }^{\circ} \mathrm{C}, 15 \mathrm{~s} ; 72{ }^{\circ} \mathrm{C}, 15 \mathrm{~s}\right.$ ), and a melting curve $\left(95^{\circ} \mathrm{C}, 10 \mathrm{~s} ; 65^{\circ} \mathrm{C}, 30 \mathrm{~s}\right.$ and $\left.95^{\circ} \mathrm{C}, 1 \mathrm{~s}\right)$. Sequence information for Ccap (sample TIUZ) was obtained from the $1 \mathrm{KP}$ project ${ }^{41}$. Reference genes for Ncfi (6phosphogluconate decarboxylating 3, 6PGDC, Ncfi_contig_3009; Sister chromatid cohesion PDS5, PDS5, Ncfi_contig_518), and Gpru and Ccap species (Eukaryotic translation initiation factor 3, EIFL3, Gpru_contig_12008; Histone deacetylase 15, HDAC15, Gpru_contig_14406) were selected from our RNA-Seq analyzes. Specific primers for IREG/ferroportin and reference genes were designed to produce amplicons of 100-200 nt (Supplementary Data 11). Relative gene expression was calculated using the primer efficiency correction method ${ }^{42}$. Two technical replicates and 3 independent biological replicates were used for each experiment.

General statistics. Sample exploration and replication of sequencing data analysis were conducted using limma $\mathrm{R}$ package ${ }^{43}$, correlation analyses were conducted by linear regression using generic stats functions of $\mathrm{R}$ software ${ }^{44}$ and Heat-map clustering was carried out using heatmap.2 function of gplots $\mathrm{R}$ package $\mathrm{e}^{45}$. 
Data availability. Raw sequence files, read count files and de novo assembled transcriptomes are available in the NCBI's Gene Expression Omnibus under the SuperSeries accession number GSE116054 or SubSeries accession numbers GSE115411 (Brassicaceae), GSE116051 (Rubiaceae New Caledonia), GSE116050 (Rubiaceae Cuba), GSE116048 (Cunoniaceae), GSE116052 (Salicaceae) and GSE116049 (Euphorbiaceae).

25. Gonneau, C. et al. Demographic history of the trace metal hyperaccumulator Noccaea caerulescens (J. Presl and C. Presl) F. K. Mey. in Western Europe. Mol. Ecol. 26, 904-922 (2017).

26. Jaffré, T., Pillon, Y., Thomine, S. \& Merlot, S. The metal hyperaccumulators from New Caledonia can broaden our understanding of nickel accumulation in plants. Front. Plant Sci. 4, 279 (2013).

27. Jestrow, B., Gutiérrez Amaro, J. \& Francisco-Ortega, J. Islands within islands: A molecular phylogenetic study of the Leucocroton alliance (Euphorbiaceae) across the Caribbean Islands and within the serpentinite archipelago of Cuba. J. Biogeogr. 39, 452-464 (2012).

28. Borhidi, A., Oviedo-Prieto, R. \& Fernández-Zequeira, M. Nuevos resultados de la revisión taxonómica de los géneros Palicourea y Psychotria (Rubiaceae, Psychotrieae) en Cuba. Acta Bot. Hung. 58, 1-48 (2016).

29. Lanquar, V. et al. Export of Vacuolar Manganese by AtNRAMP3 and AtNRAMP4 Is Required for Optimal Photosynthesis and Growth under Manganese Deficiency. Plant Physiol. 152, 1986-1999 (2010).

30. Gonzalez, D. \& Matrella, S. Nickel hyperaccumulation 'in vitro' by Leucocroton havanensis (Euphorbiaceae). Rev. del Jardín Botánico Nac. 34/35, 83-88 (2013).

31. Johnson, M. T. J. et al. Evaluating methods for isolating total RNA and predicting the success of sequencing phylogenetically diverse plant transcriptomes. PLoS One 7, e50226 (2012). 
32. Kopylova, E., Noé, L. \& Touzet, H. SortMeRNA: Fast and accurate filtering of ribosomal RNAs in metatranscriptomic data. Bioinformatics 28, 3211-3217 (2012).

33. Conesa, A. et al. Blast2GO: A universal tool for annotation, visualization and analysis in functional genomics research. Bioinformatics 21, 3674-3676 (2005).

34. Emms, D. M. \& Kelly, S. OrthoFinder: solving fundamental biases in whole genome comparisons dramatically improves orthogroup inference accuracy. Genome Biol. 16, 1-14 (2015).

35. Robinson, M. D., McCarthy, D. J. \& Smyth, G. K. edgeR: A Bioconductor package for differential expression analysis of digital gene expression data. Bioinformatics 26, 139-140 (2010).

36. Oomen, R. J. F. J. et al. Functional characterization of NRAMP3 and NRAMP4 from the metal hyperaccumulator Thlaspi caerulescens. New Phytol. 181, 637-650 (2009).

37. Curtis, M. D. \& Grossniklaus, U. A Gateway cloning vector set for high-throughput functional analysis of genes in planta. Plant Physiol. 133 (2), 462-469 (2003).

38. Schwab, R., Ossowski, S., Riester, M., Warthmann, N. \& Weigel, D. Highly specific gene silencing by artificial microRNAs in Arabidopsis. Plant Cell 18, 1121-1133 (2006).

39. Karimi, M., Inzé, D. \& Depicker, A. GATEWAY vectors for Agrobacterium-mediated plant transformation. Trends Plant Sci. 7, 193-195 (2002).

40. Lin, Y., Hassan, Z., Talukdar, S., Schat, H. \& Aarts, M. G. M. Expression of the ZNT1 zinc transporter from the metal Hyperaccumulator Noccaea caerulescens confers enhanced zinc and cadmium tolerance and accumulation to Arabidopsis thaliana. PLoS One 11, e0149750 (2016).

41. Matasci, N. et al. Data access for the 1,000 Plants (1KP) project. Gigascience 3, $1-17(2014)$.

42. Pfaffl, M. W. A new mathematical model for relative quantification in real-time RTPCR. Nucleic Acids Res. 29, 2002-2007 (2001). 
43. Ritchie, M. E. et al. Limma powers differential expression analyses for RNAsequencing and microarray studies. Nucleic Acid Res. 43, e47 (2015).

44. R Core Team. R: A language and environment for statistical computing. $R$ Foundation for Statistical Computing, Vienna, Austria. URL http://www.Rproject.org/ (2015).

45. Warnes, G. R. Gplots: Various R programming tools for plotting data. R package version 3.2.1. http://cran.r- project.org/web/packages/gplots/index.html (2015). 
Table 1. De novo assembled transcriptomes of nickel hyperaccumulators and related nonnickel accumulator species.

\begin{tabular}{|c|c|c|c|c|c|}
\hline Family/ Species & Trait* $^{*}$ & Origin & $\begin{array}{l}\text { Nbr. } \\
\text { contigs }\end{array}$ & $\begin{array}{l}\text { Assembly } \\
\text { size (Mbp) }\end{array}$ & N50 (bp) \\
\hline \multicolumn{6}{|l|}{ Brassicaceae - Noccaea } \\
\hline $\begin{array}{l}\text { Noccaea caerulescens } \\
\text { subsp. firmiensis (Ncfi) }\end{array}$ & $\mathrm{NiH}$ & France & 41,843 & 43.6 & 1,735 \\
\hline N. caerulescens Viviez (Ncviv) & $\mathrm{ZnH}$ & France & $\mathrm{nd}^{*}$ & $\mathrm{nd}^{*}$ & $\mathrm{nd}^{*}$ \\
\hline N. montana (Nmon) & NA & France & 87,243 & 48.8 & 692 \\
\hline \multicolumn{6}{|l|}{ Rubiaceae - Psychotria } \\
\hline Psychotria gabriellae (Pgab) & $\mathrm{NiH}$ & New Caledonia & 60,899 & 45.5 & 1,140 \\
\hline P. semperflorens (Psem) & NA & New Caledonia & 66,755 & 49.8 & 1,181 \\
\hline P. grandis (Pgra) & $\mathrm{NiH}$ & Cuba & 45,143 & 36.8 & 1,344 \\
\hline P. costivenia (Pcos) & $\mathrm{NiH}$ & Cuba & 46,451 & 35.1 & 1,240 \\
\hline P. clementis (Pcle) & NA & Cuba & 56,754 & 44.0 & 1,245 \\
\hline \multicolumn{6}{|l|}{ Cunoniaceae - Geissois } \\
\hline Geissois pruinosa (Gpru) & $\mathrm{NiH}$ & New Caledonia & 54,969 & 42.1 & 1,243 \\
\hline G. racemosa (Grac) & NA & New Caledonia & 58,386 & 43.7 & 1,021 \\
\hline \multicolumn{6}{|l|}{ Salicaceae - Homalium } \\
\hline Homalium kanaliense (Hkan) & $\mathrm{NiH}$ & New Caledonia & 52,634 & 40.6 & 1,259 \\
\hline H. betulifolium (Hbet) & NA & New Caledonia & 49,962 & 39.0 & 1,294 \\
\hline \multicolumn{6}{|l|}{ Euphorbiaceae - Adelieae tribe } \\
\hline Leucocroton havanensis (Lhav) & $\mathrm{NiH}$ & Cuba & 58,990 & 44.4 & 1,206 \\
\hline Lasiocroton microphyllus (Lmic) & NA & Cuba & 52,421 & 39.8 & 1,244 \\
\hline
\end{tabular}

* $\mathrm{NiH}$ (Ni hyperaccumulator), $\mathrm{ZnH}$ (Zn hyperaccumulator), NA (non-accumulator), nd (nondetermined) 
C
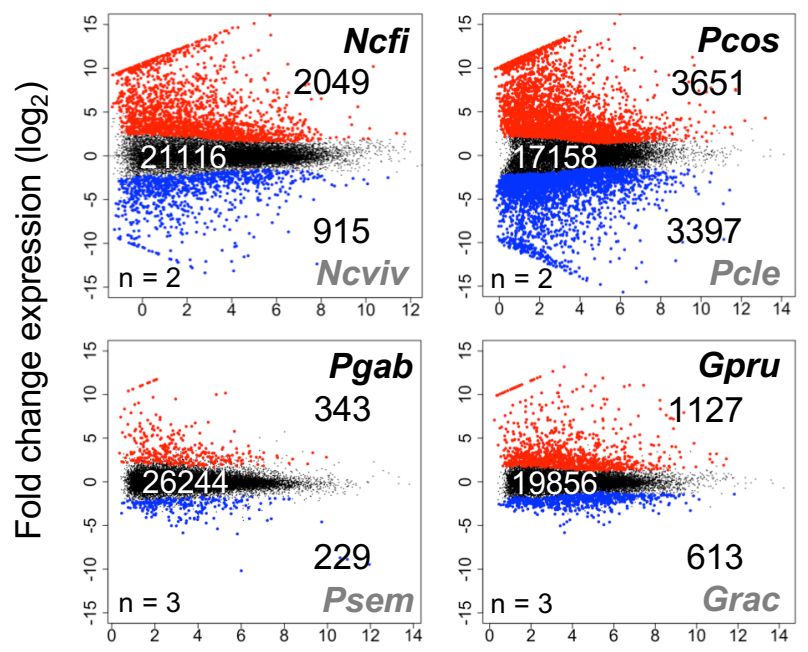

Average gene expression ( $\left.\log _{2} \mathrm{CPM}\right)$

b

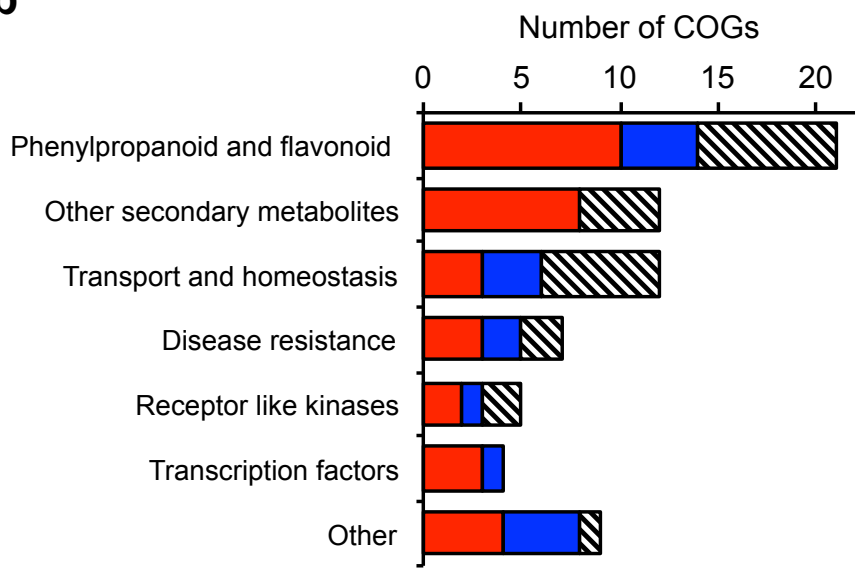

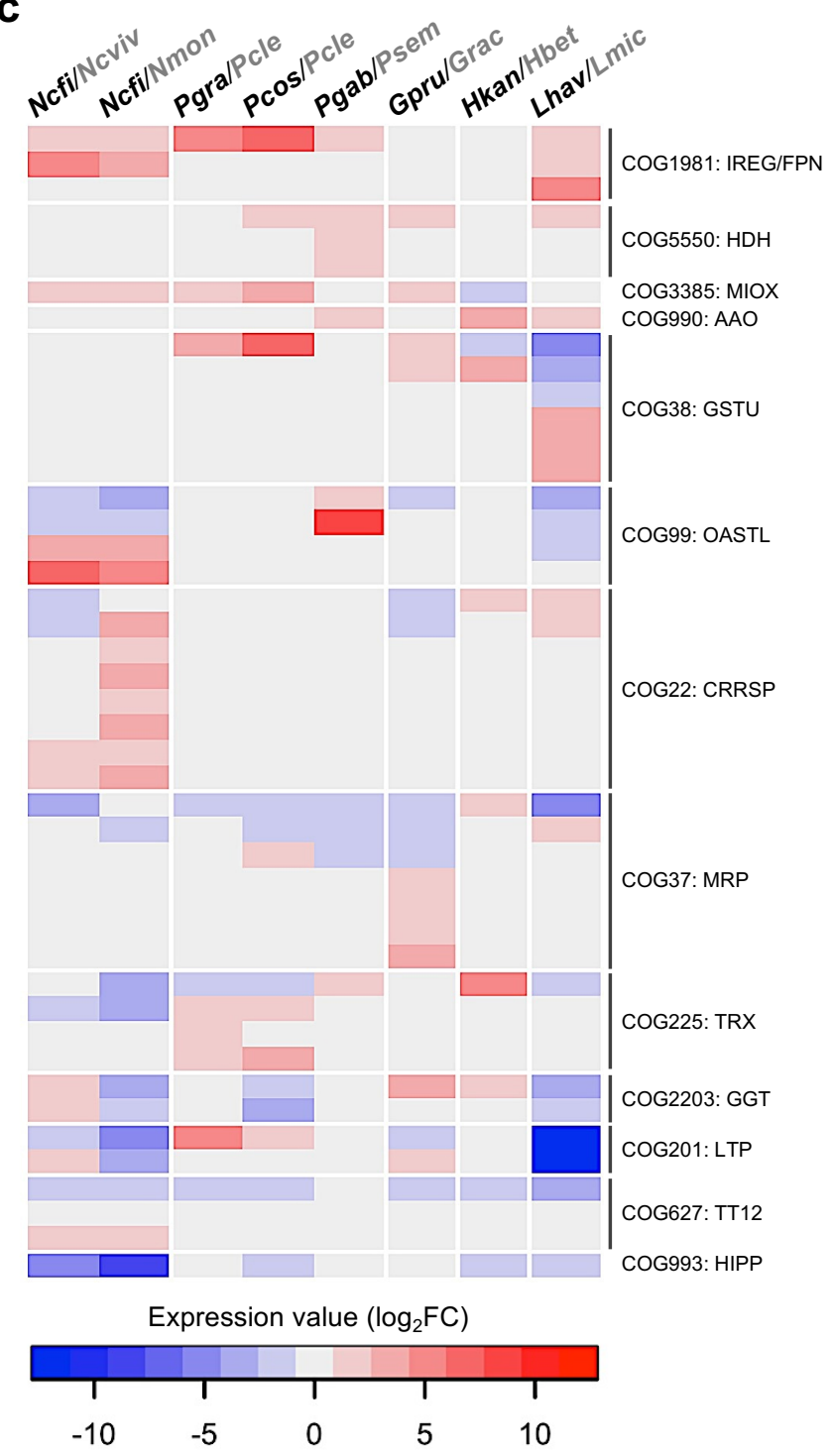

Figure 2. Cross-family comparison reveals orthologous genes convergently associated with nickel hyperaccumulation. (a) Cross-species comparative transcriptomic reveals Differential Expressed (DE) genes between nickel hyperaccumulator species (black) and related non-nickel accumulators (grey). Genes (contigs) are plotted according to their mean level of expression (x-axis) and their differential expression ( $y$-axis) in the pair of species. The numbers of significant DE (red and blue dots) and non DE genes are indicated $\left(\log _{2} \mathrm{FC}>1\right.$, $F D R<0.05$ ).(b) Distribution of the 71 selected Cluster of Orthologous Groups (COGs) associated with nickel hyperaccumulation according to their predicted function and their level of expression in hyperaccumulators: Red represents COGs containing DE genes more expressed in nickel hyperaccumulators belonging to at least 3 distinct plant families, the blue color for COGs containing DE genes less expressed in nickel hyperaccumulators, and hatched for COGs containing DE genes both more and less expressed in nickel hyperaccumulators. (c) Heat-map of COGs containing genes related to transport and homeostasis function that are differentially expressed in nickel hyperaccumulators compared to related non-accumulators. Abbreviations: IREG/FPN (Iron Regulated/ Ferroportin), HDH (Histidinol Dehydrogenase ), MIOX (Myo-Inositol Oxygenase), AAO (Ascorbate Oxidase), GSTU (Glutathione-s-transferase Tau), OASTL (Cysteine Synthase), CRRSP (Cysteine-rich Repeat Secretory Protein), MRP (Multidrug Resistance Associated Protein/ABCC), TRX (Thioredoxin-H-type Protein), GGT (Gamma-Glutamyltransferase), LTP (Lipid Transfer Protein), TT12 (Mate Transparent Testa12), HIPP (Heavy Metal-associated Isoprenylated Plant Protein). 


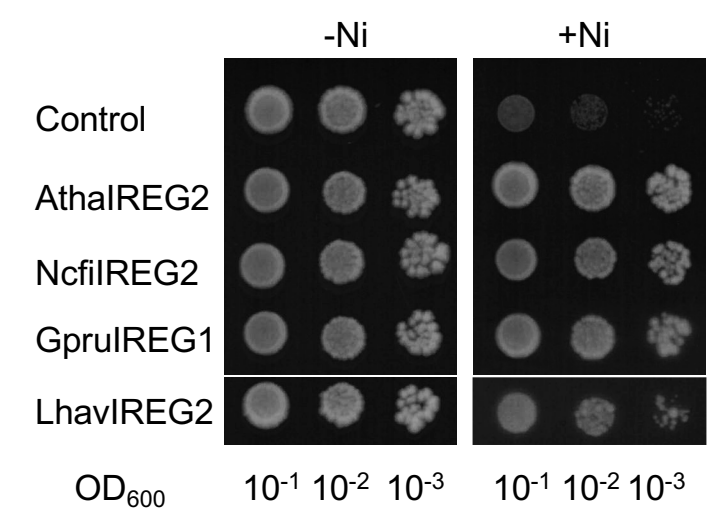

b

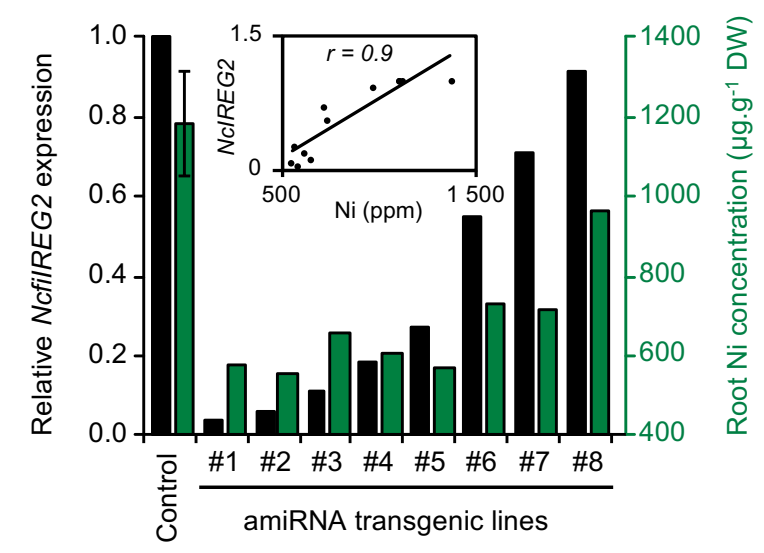

C

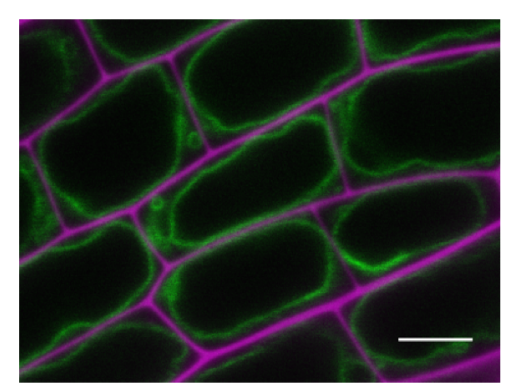

Figure 3. IREG/Ferroportin transporters have a conserved role in nickel accumulation. (a) Expression of plant IREG/Ferroportin transporters increases nickel tolerance in yeast. Yeast cells expressing IREG/Ferroportin transporters cloned from A. thaliana, $N$. caerulescens, $G$. pruinosa and $L$. havanensis were plated at different dilutions on a medium containing a toxic concentration of nickel for the control line (transformed with pDR195 vector). (b) Silencing of the IREG/Ferroportin transporter IREG2 in roots of the nickel hyperaccumulator $N$. caerulescens subsp. firmiensis reduces nickel accumulation. The expression of NcIREG2 was quantified by RTqPCR (black bars) in 8 amiRNA transgenic lines and control lines transformed with the pK7WG2D vector $(n=3)$ growing in presence of $37.5 \mu \mathrm{M} \mathrm{NiCl} 2$ for 4 weeks. NcIREG2 expression was corrected using Nc6PGDC as reference gene and normalized to 1 for the expression in control lines. Nickel concentration was measured in parallel in the roots of the same lines by MP-AES (green bars). The insert presents the Pearson's linear correlation between NcfilREG2 expression and nickel accumulation ( $P$-value $<0.001)$. (c) NcfilREG2 localizes on the vacuolar membrane in $N$. caerulescens cells. Confocal picture of a transgenic line expressing NcfilREG2-GFP (green) in root cells. Cell wall was stained with propidium iodide (magenta). The scale bar correspond to $5 \mu \mathrm{m}$. 